\title{
Sistemas de gradação histológica em queilites actínicas: revisão de literatura
}

Histological grading systems in actinic cheilitis: literature review

Sistemas de gradación histológica en queilitis actínica: revisión de la literatura

Elton Fernandes BARROS ${ }^{1}$

Livian Isabel de Medeiros CARVALHO

Itainar Henriques CARVALHO ${ }^{1}$

Amanda Pereira FERRAZ ${ }^{2}$

Hellen Bandeira de Pontes SANTOS ${ }^{3}$

${ }^{1}$ Aluno de Iniciação Científica - Curso de Odontologia - Faculdades Nova Esperança - FACENE- 58067-695- João Pessoa - PB - Brasil

${ }^{2}$ Graduanda-Curso de Odontologia-UFPB- 58051-900- João Pessoa-PB-Brasil

${ }^{3}$ Programa de Pós-Graduação em Saúde da Família - Faculdades Nova Esperança - FACENE- 58067-695- João Pessoa - PB - Brasil

\section{Resumo}

Introdução: A queilite actínica (QA) é uma desordem potencialmente maligna associada à exposição crônica à luz solar como principal fator etiológico. A QA tem como principal sítio de acometimento o vermelhão do lábio inferior, apresentando características clínicas marcantes. Porém, a análise histopatológica desta lesão merece um enfoque especial pelas características bastante expressivas para o potencial de malignidade. Assim, para essa análise, são elencados dois sistemas principais: o sistema da Organização Mundial da Saúde (OMS), e o binário. Objetivo: Realizar uma revisão da literatura para demonstrar os pontos avaliados e o valor preditivo dos sistemas de gradação histológica em QA. Metodologia: Trata-se de um estudo de revisão bibliográfica, utilizando artigos da base de dados da SCIELO e PUBMED, encontrados com o uso dos descritores: "actinic cheilitis", "WHO system", "binary system", "oral potentially malignant disorders", "histological features in actinic cheilitis", fazendo uso do operador booleano "AND". Conclusão: Diante da revisão realizada, percebeu-se a importância da adoção dos sistemas de gradação histológica da OMS, e o binário para uma análise minuciosa de lesões de QA, que apresentam potencial de malignidade. Ademais, constatou-se o favorecimento do sistema binário na redução da subjetividade entre os patologistas quanto à gradação das avaliações histopatológicas.

Descritores: Queilite; Patologia Bucal; Neoplasias Labiais; Classificação; Diagnóstico.

\section{Abstract}

Introduction: Dental attention is of paramount importance for parents to raise awareness about their child's oral health from the first months of life, emphasizing information on oral hygiene, diet, and parafunctional habits. Objectives: The aim of this study was to evaluate the knowledge of parents and/or guardians of children in early childhood regarding the daily conduct of hygiene of their children. Methodology: For this, a questionnaire was applied, with objective questions about the knowledge of oral hygiene habits in relation to your child in five daycare centers of children 0-4 years old. Data were tabulated using the Microsoft Excel (® 2016 software and the results obtained by descriptive and quantitative analysis. Results: Parents and/or guardians interviewed, all reported to know the importance of the primary dentition and $97 \%$ knew what it was dental caries disease. As for brushing $98 \%$ of children do daily, however, only $55 \%$ of these children's parents help with brushing and only $34 \%$ perform three times a day. All respondents claimed to be aware of the importance of primary teeth and 50\% answered that the child should go to the dentist for the first time when the first tooth that erupts in the oral cavity. Conclusion: Thus, it is concluded that the knowledge of parents and/or guardians about the importance of oral hygiene practices are fundamental, where simple preventive practices allow the development of the healthy primary dentition, however, this information needs to be passed to those responsible.

Descriptors: Oral Health; Dental Care; Oral Hygiene.

\section{Resumen}

Introducción: la atención dental es de suma importancia para la conciencia de los padres sobre la salud bucal de sus hijos desde los primeros meses de vida, destacando información sobre higiene bucal, dieta y hábitos parafuncionales. Objetivos: Evaluar el conocimiento de los padres y / o tutores de los niños en la primera infancia con respecto a la conducta diaria de higiene de sus hijos. Metodología: se aplicó un cuestionario con preguntas objetivas sobre el conocimiento de los hábitos de higiene bucal en relación con su hijo en cinco guarderías para niños de 0 a 4 años. Los datos se tabularon con el software Microsoft Excel@ 2016 y los resultados se obtuvieron mediante un análisis descriptivo y cuantitativo. Resultados: De los padres y / o tutores entrevistados, todos informaron conocer la importancia de la dentición decidua y el $97 \%$ sabían era lo que era enfermedad de caries dental. En cuanto al cepillado, el 98\% de los niños lo hacen a diario, sin embargo, solo el 55\% de estos niños son ayudan por estos padres e/o tutores cepillado y solo el $34 \%$ realiza tres veces al día. Todos los encuestados dijeron que eran conscientes de la importancia de la dentición decidua y el $50 \%$ respondió que el niño debería ir al dentista por primera vez cuando el primer diente erupcione en la cavidad oral. Conclusión: Por lo tanto, se concluye que el conocimiento de los padres y / o tutores sobre la importancia de la práctica de higiene oral es fundamental, donde las prácticas simples de prevención permiten el desarrollo de una dentición decidua saludable, sin embargo, esta información debe transmitirse a responsable.

Descriptores: Salud Bucal; Cuidado Dental; Higiene Bucal.

\section{INTRODUÇÃO}

A queilite actínica (QA) é uma condição potencialmente maligna que ocorre com maior frequência no vermelhão do lábio inferior, uma região que marca a transição de mucosa para o tecido cutâneo, apresentando uma camada delgada de epitélio $^{1-6}$. Esta condição apresenta risco iminente de proliferação celular desproporcional, podendo evoluir para um carcinoma de células escamosas de lábio. A etiologia da QA está principalmente relacionada à exposição crônica à radiação ultravioleta, indutora de alterações genotípicas e fenotípicas nas células epiteliais, as quais podem resultar em displasias epiteliais ${ }^{3,5,7}$.
De acordo com o aspecto clínico, a QA pode se manifestar de forma aguda, quando há exposição intensa ao sol em breve espaço de tempo, sendo

descrita pela presença de edema e eritema leves, bem como fissura e úlceras graves; ou crônica, quando há exposição à radiação ultravioleta de forma intensa e por um longo período ${ }^{1,2,8}$. Os pacientes com QA crônica geralmente demonstram lábios ressecados, fissuras, manchas leucoplásicas e áreas edemaciadas ${ }^{1,2,8}$. Há uma maior prevalência desta lesão em indivíduos do sexo masculino e de cor de pele clara, pois existe uma menor quantidade de melanina, um pigmento natural que confere proteção ao efeito carcinogênico da radiação ultravioleta. 
Além disso, há um maior acometimento em indivíduos que trabalham ao ar livre, como agricultores e marinheiros ${ }^{1,2,5-10}$. Contudo, clinicamente, não se consegue predizer o potencial de malignidade desta lesão, necessitando-se da execução da biópsia incisional para posterior análise histopatológica ${ }^{1,11}$.

Histologicamente, esta lesão pode demonstrar diversas alterações epiteliais, como: hiperplasia, acantose ou atrofia do epitélio, hiperceratinização, atipia celular, aumento de atividade mitótica, presença ou ausência de displasia; assim como, no tecido conjuntivo, a presença de elastose solar, que se trata da degeneração basofílica de fibras colágenas ${ }^{1,2,6-8,11}$. Além disso, é perceptível a presença da associação entre a displasia e o grau de infiltrado inflamatório ${ }^{2,12}$. Segundo a maioria dos estudos, há uma expressão marcante da incidência de displasia epitelial (DE) em QAs, o que evidencia a necessidade de análise das características microscópicas através do exame anatomopatológico, sabendo-se que é notável uma relação entre o aumento do potencial de malignidade de acordo com o grau de displasia ${ }^{1,2,3,10}$.

O termo displasia é aplicado quando uma desordem nas características arquiteturais é associada a uma atipia celular, ou seja, a displasia epitelial é marcada pelo conjunto de alterações arquiteturais e citológicas nos estratos do epitélio ${ }^{13}$. Assim, através da análise microscópica, é possível constatar a presença ou a ausência de displasia, podendo identificar também a progressão; realizando assim, a gradação histológica ${ }^{1,14}$.

De acordo com a análise da literatura, é possível elencar dois principais sistemas de gradação histológica utilizados para um diagnóstico preciso da severidade da QA: 1) sistema de classificação da OMS, categorizado em graus leve, moderado e severo, comumente utilizado e se baseia na análise das alterações arquiteturais e citológicas do epitélio; 2) sistema binário, proposto por Kujan et al. ${ }^{15}$, que se embasa nas alterações arquiteturais e citológicas estabelecidas pela OMS, mas estabelece uma classificação em dois grupos, baixo risco e alto risco $^{3,10,13-16}$.

Dessa forma, este trabalho objetivou realizar uma revisão de literatura sobre os sistemas de gradação histológica da OMS e o binário em QAs como forma de demonstrar as características inerentes, comparações, e a relevância da utilização como forma de obter um diagnóstico precoce, principalmente, pelo caráter de potencial de malignidade, e uma conduta clínica adequada.

\section{MATERIAL E MÉTODO}

Para a elaboração desta revisão de literatura, utilizaram-se como bases de dados PubMed e Scielo, buscando publicações eletrônicas, através dos seguintes termos: "actinic cheilitis", "WHO system", "binary system", "potentially malignant disorders", "histological features in actinic cheilitis", fazendo uso de operador booleano "AND". Assim, procurouse fazer a seleção de publicações científicas para a análise de relevância para a construção desta revisão, de acordo com alguns critérios de inclusão: $1^{\circ}$ ) artigos que evidenciassem a abordagem das características clínicas e histopatológicas da QA, $2^{\circ}$ ) artigos que demonstrassem os sistemas de gradação da OMS e o sistema binário, e $3^{\circ}$ ) artigos que correlacionassem os sistemas da OMS e o binário, debatendo a utilização e suas características. Dessa maneira, de acordo com os critérios, foram selecionados 30 artigos; sendo, após a análise, escolhida uma quantidade de 22 trabalhos.

RESULTADOS

- Sistema de gradação da OMS

O sistema de gradação da Organização Mundial da Saúde (OMS), proposto em 2005, consiste em uma classificação, como resultado da análise de alguns critérios das alterações arquiteturais e citológicas do epitélio (tabela 1), de displasia epitelial em graus: leve, moderado, severo, e carcinoma in situ (Tabela 2) $3,13,14,18,19$. Este sistema é amplamente utilizado, porém a sua reprodutibilidade tem sido questionada, especialmente devido ao fato de possuir muitas categorias, o que dificulta a objetividade do patologista ${ }^{3,14}$. Em 2017, a OMS atualizou seu sistema de gradação $^{20}$, tratando a categoria "carcinoma in situ" como sinônimo de displasia severa.

Neste sistema, a gradação é definida de acordo com o acometimento das camadas do epitélio pelas alterações arquiteturais e citológicas: displasia leve (alterações arquiteturais restritas ao terço inferior do epitélio com atipia celular mínima), moderada (extensão das alterações arquiteturais e citológicas para o terço médio do epitélio), e severa (alterações arquiteturais e atipia celular além do terço médio do epitélio) $)^{3,13,14}$. Entretanto, de acordo com alguns estudos, este sistema apresenta um estimável grau de subjetividade de diagnóstico entre os patologistas pela existência de uma categoria intermediária ${ }^{1,3,14,15,18,21}$.

\begin{tabular}{|c|c|c|c|}
\hline \multicolumn{2}{|l|}{ Arquitetura } & \multicolumn{2}{|r|}{ Citologia } \\
\hline \multicolumn{2}{|c|}{$\begin{array}{ll}\text { - } & \text { Cristas epiteliais em formato de gota } \\
\text { Aumento do número de figuras } \\
\text { mitóticas } \\
\text { - } & \text { Mitoses superficiais } \\
\text { Disqueratose } \\
\quad \text { Pérolas de ceratina dentro das cristas } \\
\text { epiteliais }\end{array}$} & 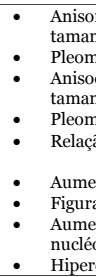 & $\begin{array}{l}\text { cleose (variação anormal no } \\
\text { do núcleo) } \\
\text { fismo nuclear } \\
\text { sse (variacãa anormal no } \\
\text { da célula) } \\
\text { fismo celular } \\
\text { núcleo-citoplasma aumentada } \\
\text { do tamanho nuclear } \\
\text { nitóticas atípicas } \\
\text { do número e tamanho de } \\
\text { masia }\end{array}$ \\
\hline \multicolumn{4}{|l|}{ Fonte: El-Naggar et al. ${ }^{20}$} \\
\hline \multicolumn{4}{|l|}{ Tabela 2. Sistema OMS } \\
\hline Leve & \multicolumn{2}{|c|}{ Moderado } & Severo \\
\hline $\begin{array}{l}\text { Alterações limitadas ao } \\
\text { terço inferior do } \\
\text { epitélio }\end{array}$ & \multicolumn{2}{|c|}{$\begin{array}{l}\text { Alterações restritas aos } \\
\text { dois terços inferiores } \\
\text { do epitélio }\end{array}$} & $\begin{array}{l}\text { Mudanças ultrapassando } \\
\text { o terço médio do epitélio }\end{array}$ \\
\hline
\end{tabular}




\section{- Sistema binário}

O sistema de gradação binário, proposto por Kujan et al. ${ }^{15}$, instituiu uma nova formulação para a realização da classificação da displasia epitelial para a redução da subjetividade inerente à gradação no sistema da OMS, trazendo apenas dois graus: baixo risco, ou alto risco de transformação maligna, baseando-se nos mesmos critérios de alterações arquiteturais e citológicas no diagnóstico de displasia do sistema da OMS (Tabela 1). Sendo assim, este sistema realiza a gradação referente às alterações arquiteturais e citológicas identificadas em dois graus: baixo risco, apresentando menos que quatro (4) alterações arquiteturais ou menos que cinco (5) citológicas; e alto-risco, exibindo até quatro (4) mudanças arquiteturais e cinco (5) citológicas

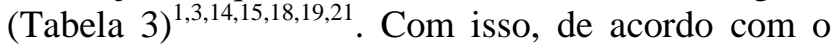
ponto de vista clínico, conseguiu-se obter uma concordância considerável entre os patologistas, por meio da redução da subjetividade proposta por este sistema ${ }^{1,14,15,19,21}$.

Tabela 3. Sistema binário

\begin{tabular}{c|c}
\hline Baixo risco & Alto risco \\
\hline $\begin{array}{c}\text { Menos que quatro (4) alterações } \\
\text { arquiteturais ou menos que cinco (5) } \\
\text { citológicas }\end{array}$ & $\begin{array}{c}\text { Até quatro (4) mudanças arquiteturais e } \\
\text { cinco (5) citológicas }\end{array}$ \\
\hline
\end{tabular}

Fonte: Kujan et al. ${ }^{15}$

\section{DISCUSSÃO}

A queilite actínica (QA) é uma desordem potencialmente maligna, com incidência maior no vermelhão do lábio inferior. Tem sido demonstrado que alguns casos de QA podem estar associados à presença de displasia epitelial ${ }^{1,2,3,7,9,10,14}$. O grau da displasia epitelial pode ser classificado de acordo com sistemas de gradação histopatológica, os quais se destacam o sistema de gradação da OMS e o binário, que oferecem instrumentos imprescindíveis para a verificação da potencialidade da lesão, visto que pode haver transformação maligna ${ }^{3,13-15,19}$. Diante disso, este trabalho realizou uma revisão da literatura sobre os sistemas de gradação histológica da QA, enfatizando sua possível relevância clínica, e como forma de predição de malignidade.

De acordo com Warnakulasuriya et al. ${ }^{11}$, o diagnóstico provisório de QA pode ser conferido de acordo com as características clínicas, mas para diagnóstico definitivo, a biópsia é essencial. Na biópsia, a avaliação histopatológica é o "padrão ouro" de diagnóstico para desordens potencialmente malignas. Por sua vez, o os sistemas de gradação fornecem o grau de displasia epitelial, o que orienta o clínico para o prognóstico e o manejo mais apropriado da lesão ${ }^{1,11,14,17,21}$. Dessa maneira, a biópsia incisional é muito importante para lesões com aspectos clínicos mais severos, com intuito de prevenir a transformação maligna; como também, torna-se fundamental o acompanhamento periódico de pacientes com $\mathrm{QA}^{1}$.
Sendo assim, para a classificação da displasia epitelial em QA, utiliza-se o sistema de gradação da OMS, que se apresenta em três graus: leve, moderado e severo, conferindo um diagnóstico de acordo com as alterações citológicas e arquiteturais, estabelecido pelo nível de acometimento dos estratos do epitélio ${ }^{3,13,14,18}$. Contudo, mesmo sendo bastante utilizado, é um sistema que apresenta um estimável grau de subjetividade de diagnóstico entre os patologistas pela existência de uma categoria intermediária ${ }^{3,14,18,21}$. Assim, Kujan et al. ${ }^{15}$ propuseram um novo sistema, conhecido como sistema binário, baseado nos mesmos critérios definidos pela classificação da OMS, de mudanças arquiteturais e citológicas, que estabelece dois graus: baixo risco e alto risco, conferindo o diagnóstico a partir da contagem das alterações presentes. Dessa maneira, o sistema binário apresentando menos categorias auxilia na redução da subjetividade entre os patologistas $3,14,15,19$.

Analisando o sistema binário em relação ao da OMS, consolidado como o utilizado mundialmente, há uma correlação positiva entre os sistemas para avaliação histopatológica da QA de acordo com Câmara et al. ${ }^{14}$, Pilati et al. ${ }^{3}$ e Kujan et al. ${ }^{15}$. Porém, Nagata et al. ${ }^{16}$ demonstraram uma discrepância entre os sistemas mesmo sendo baseados nos mesmos critérios. Ademais, com a subjetividade e fácil reprodutibilidade do sistema da OMS, Lopes et al. ${ }^{1}$, Shubhasini et al. ${ }^{21}$, Câmara et al. ${ }^{14}$, Pilati et al. $^{3}$, Kujan et al. ${ }^{15}$ demonstraram que o sistema binário é importante, reduzindo a subjetividade para ajudar os patologistas em casos de pouca concordância e na existência de dúvidas. Contudo, sendo a displasia epitelial um processo evolutivo, é de extrema necessidade ressaltar a dependência ao observador, ou seja, o profissional, independente dos sistemas utilizados ${ }^{3,14}$.

Segundo Lopes et al. ${ }^{1}$, com a análise de 161 casos de pacientes diagnosticados com QA, houve uma prevalência da lesão em lábio inferior $(97,5 \%)$, com características clínicas de placas brancas $(33,6 \%)$, de vermelhas e brancas $(26,6 \%)$, e de vermelhas $(25,9 \%)$, sendo as biópsias realizadas em 83 pacientes, onde a gradação resultou em 50 casos $(60,2 \%)$ de baixo risco, e 33 casos $(39,8 \%)$ de alto risco, com os critérios do sistema binário. Além disso, o estudo demonstrou não haver relação entre as caraterísticas clínicas e demográficas com o risco histopatológico de transformação maligna das lesões, ratificando a necessidade da biópsia para diagnosticar e adotar o melhor manejo terapêutico ${ }^{1}$.

Câmara et al. ${ }^{14}$ analisaram 107 casos de QA de acordo com os sistemas da OMS e binário. No sistema da OMS, obtiveram um resultado de maior prevalência de displasia epitelial leve (48 casos), seguido de moderada (35 casos), e severa (11 casos); em treze casos, não foi constatada displasia. Por sua 
vez, no sistema binário, 69 casos foram classificados como de baixo risco, e 38 casos em alto risco de transformação maligna. Além disso, os casos gradados no sistema da OMS como sem DE e displasia leve foram reunidos no grupo de baixo risco do sistema binário; os com $\mathrm{DE}$ grave foram agrupados como alto risco. Já os casos de DE moderada no sistema da OMS foram distribuídos quase igualmente entre os grupos de baixo risco (17 casos) e de alto risco (18 casos no sistema binário, o que demonstra o grande desafio dos patologistas para gradação no sistema da OMS com a presença do grau moderado $^{14}$.

Pilati et al. ${ }^{3}$ por meio da análise de 58 casos de QA, de acordo com o sistema binário, 36,2\% das lesões foram graduadas como de baixo risco e $63,8 \%$ de alto risco. Já, seguindo o sistema da OMS, 18 casos foram graduados histopatologicamente como DE leve, 31 como DE moderada, e 8 como DE severa, sendo um caso classificado como sem displasia. Com isso, $87,5 \%$ das DE graves e $77,4 \%$ das moderadas da OMS foram classificadas como de alto risco no sistema binário. Com isso, com a redução de subjetividade pelo sistema binário, os resultados encontrados sugeriram esse sistema como de mais fácil diagnóstico ${ }^{3}$.

A partir da classificação dos graus de displasia epitelial com base nos sistemas de gradação OMS e binário, é possível predizer o potencial de malignidade da QA, enfatizando que, quanto mais avançado o grau, mais provável é a transformação maligna ${ }^{1,2,14,15,19}$. Além disso, há associação entre a presença de inflamação e a progressão da displasia $^{2,12}$. Sendo assim, com as lesões prémalignas orais representando um notável fator de risco para desenvolvimento de carcinoma escamocelular ${ }^{22}$, a determinação do grau da displasia é imprescindível para a adoção da conduta terapêutica adequada e o manejo do paciente, o que faz dos sistemas de gradação ferramentas essenciais no auxílio de predizer o prognóstico ${ }^{1,3,8,13,17,19,21}$.

\section{CONCLUSÃO}

A elevada incidência de displasia epitelial em lesões de QA, que já é uma desordem com potencial de malignidade, demonstra, através dos sistemas de gradação histopatológica, que, quanto mais avançado o nível de displasia, maior a probabilidade para a transformação maligna, enfatizando a expressão significativa dos sistemas de gradação da OMS, e binário.

Ademais, é importante a ênfase em que o sistema da OMS, que classifica as displasias em três graus: leve, moderado e severo, apresenta grande significância no diagnóstico de displasias. Mas, diante da subjetividade e fácil reprodutibilidade do sistema da OMS, o sistema binário, que grada as displasias em apenas dois graus: baixo risco e alto risco evidenciam a redução da subjetividade entre os patologistas, o que aumenta a concordância. Mas também, apesar das limitações dos sistemas, existe uma forte dependência do observador para gradação histopatológica das lesões, o que influencia no discernimento do grau de displasia nos sistemas, que é responsável por conduzir a correta adoção da abordagem terapêutica, e a intervenção precoce diante de uma transformação maligna.

\section{REFERÊNCIAS}

1. Lopes MLDS, Silva Junior FLS, Lima KC, Oliveira PT, Silveira EJD. Clinicopathological profile and management of 161 cases of actinic cheilitis. An Bras Dermatol. 2015;90(4):347-50.

2. Arnaud RR, Soares MSM, Paiva MAF, Figueiredo CRLV, Santos MGC, Lira CC. Queilite actínica: avaliação histopatológica de 44 casos. Rev Odontol UNESP. 2014;43(6):384-89.

3. Pilati S, Bianco BC, Vieira D, Modolo F. Histopathologic features in actinic cheilitis by the comparison of grading dysplasia systems. Oral Dis. 2017;23(2):219-24.

4. Maia HCM, Pinto ANS, Pereira JS, Medeiros AMC, Silveira EJD, Miguel MCC. Lesões orais potencialmente malignas: correlações clínicopatológicas. Einstein. 2016;14(1): 35-40.

5. Savage NW, McKay C, Faulkner C. Actinic cheilitis in dental practice. Aust Dent J. 2010; 55(Suppl 1):78-84.

6. Vieira RA, Minicucci EM, Marques ME, Marques SA. Actinic cheilitis and squamous cell carcinoma of the lip: clinical, histopathological and immunogenetic aspects. An Bras Dermatol. 2012; 87(1):105-14.

7. Dancyger A, Heard V, Huang B, Suley C, Tang $\mathrm{D}$, Ariyawardana A. Malignant transformation of actinic cheilitis: A systematic review of observational studies. J Investig Clin Dent. 2018; 9(4):e12343.

8. de Santana Sarmento DJ, da Costa Miguel MC, Queiroz LM, Godoy GP, da Silveira EJ. Actinic cheilitis: clinicopathologic profile and association with degree of dysplasia. Int J Dermatol. 2014; 53(4):466-72.

9. Wood NH, Khammissa R, Meyerov R, Lemmer J, Feller L. Actinic cheilitis: a case report and a review of the literature. Eur J Dent. 2011; 5(1):101-6.

10. Mello FW, Melo G, Modolo F, Rivero ER. Actinic cheilitis and lip squamous cell carcinoma: Literature review and new data from Brazil. J Clin Exp Dent. 2019;11(1):e62-9.

11. Warnakulasuriya S, Johnson NW, van der Waal I. Nomenclature and classification of potentially malignant disorders of the oral mucosa. J Oral Pathol Med. 2007;36(10):575-80.

12. Paiva MAF, Soares MSM, Figueiredo CRLV, 
Luna AH, Oliveira VEN, Brasil Júnior O. Associação entre displasia e inflamação em queilite actínica. J Bras Patol Med Lab. 2012; 48(6):455-58.

13. Warnakulasuriya $\mathrm{S}$, Reibel $\mathrm{J}$, Bouquot $\mathrm{J}$, Dabelsteen E. Oral epithelial dysplasia classification systems: predictive value, utility, weaknesses and scope for improvement. J Oral Pathol Med. 2008;37(3):127-33.

14. Câmara PR, Dutra SN, Takahama Júnior A, Fontes K, Azevedo RS. A comparative study using WHO and binary oral epithelial dysplasia grading systems in actinic cheilitis. Oral Dis. 2016; 22(6):523-9.

15. Kujan O, Oliver RJ, Khattab A, Roberts SA, Thakker N, Sloan P. Evaluation of a new binary system of grading oral epithelial dysplasia for prediction of malignant transformation. Oral Oncol. 2006;42(10):987-93.

16. Nagata G, Santana T, Queiroz A, Caramez RH, Trierveiler M. Evaluation of epithelial dysplasia adjacent to lip squamous cell carcinoma indicates that the degree of dysplasia is not associated with the occurrence of invasive carcinoma in this site. $J$ Cutan Pathol. 2018. doi: 10.1111/cup.13270.

17. Mello FW, Miguel AFP, Dutra KL, Porporatti AL, Warnakulasuriya S, Guerra ENS, Rivero ERC. Prevalence of oral potentially malignant disorders: A systematic review and meta-analysis. J Oral Pathol Med. 2018;47(7):633-40.

18. Izumo T. Oral premalignant lesions: from the pathological viewpoint. Int $\mathbf{J}$ Clin Oncol. 2011;16(1):15-26.

19. Kujan O, Khattab A, Oliver RJ, Roberts SA, Thakker N, Sloan P. Why oral histopathology suffers inter-observer variability on grading oral epithelial dysplasia: an attempt to understand the sources of variation. Oral Oncol. 2007; 43(3):224-31.

20. El-Naggar AK, Chan JKC, Grandis JR, Takata T, Slootweg PJ. World Health Organization Classification of Head and Neck Tumours. WHO/IARC Classification of Tumours 2017; 4th ed. Lyon, France: IARC Press.

21. R SA, B N P, Hegde U, K U, G S, G K, Sil S. Inter- and Intra-Observer Variability in Diagnosis of Oral Dysplasia. Asian Pac J Cancer Prev. 2017;18(12):3251-54.

22. Olinici D, Cotrutz CE, Mihali CV, Grecu VB, Botez EA, Stoica L, Onofrei P, Condurache O, Dimitriu DC. The ultrastructural features of the premalignant oral lesions. Rom J Morphol Embryol. 2018;59(1):243-48.

\section{CONFLITO DE INTERESSES}

Os autores declaram não haver conflitos de interesse.

\section{AUTOR PARA CORRESPONDÊNCIA}

\section{Hellen Bandeira de Pontes Santos}

Departamento de Odontologia

Faculdades Nova Esperança - FACENE

Avenida Frei Galvão,12- Gramame

58067-695- João Pessoa - PB, Brasil.

E-mail: hellenbps@ hotmail.com
Submetido em 04/01/2020

Aceito em 13/06/2020 